\title{
Non-uniform HOC scheme for the 3D convection-diffusion equation
}

\author{
Rabab Ahmed Shanab, Laila Fouad Seddek, Salwa Amin Mohamed \\ Department of Engineering Mathematics, Faculty of Engineering, Zagazig University, Egypt.
}

Email address:

rabab_shanab@yahoo.com (R. A. Shanab), laila.f.seddek@gmail.com(L. F. Seddek), s_a_mohamed@hotmail.com(S. A. Mohamed)

\section{To cite this article:}

Rabab Ahmed Shanab, Laila Fouad Seddek, Salwa Amin Mohamed. Non-uniform HOC Scheme for the 3D Convection-Diffusion Equation Applied and Computational Mathematics. Vol. 2, No. 3, 2013, pp. 64-77. doi: 10.11648/j.acm.20130203.11

\begin{abstract}
In this paper, we extend the work of Kalita et al. [11] to solve the steady 3D convection-diffusion equation with variable coefficients on non-uniform grid. The approach is based on the use of Taylor series expansion, up to the fourth order terms, to approximate the derivatives appearing in the 3D convection diffusion equation. Then the original convection-diffusion equation is used again to replace the resulting higher order derivative terms. This leads to a higher order scheme on a compact stencil (HOC) of nineteen points. Effectiveness of this method is seen from the fact that it can handle the singularity perturbed problems by employing a flexible discretized grid that can be adapted to the singularity in the domain. Four difficult test cases are chosen to demonstrate the accuracy of the present scheme. Numerical results show that the fourth order accuracy is achieved even though the Reynolds number $(\mathrm{Re})$ is high.
\end{abstract}

Keywords: 3D Convection-Diffusion Equation, Variable Coefficient, Fourth Order Compact Scheme, Non-Uniform Grids, Algebraic Multigrid

\section{Introduction}

We are concerned with numerical solution methods for solving convection-diffusion equation as it plays an important role in computational fluid dynamics (CFD). In the last two decades, a variety of specialized techniques were developed based on high order compact (HOC) finite difference (FD) schemes, which are computationally efficient. Several authors developed a number of fourthorder compact (4OC) finite difference schemes for convection diffusion equations on uniform grids for two dimensional space [1-3] and three dimensional space [47].These schemes have good numerical stability and provide high accuracy approximations for smooth problems. However, for singularly perturbed problems, if uniform grids are employed, the grids have to be refined over all computational domains. That leads to expensive and wasteful computations. Hence a non-uniform grid discretization is used to solve these problems by making grid points concentrate in the regions of singularity. One practice of non-uniform grid discretization is achieved with different mesh size in $\mathrm{x}-\mathrm{y}$ - and $\mathrm{z}$-directions however the grid is still uniform in each of these directions. Using this approach, Ge [8] solved the 3D Poisson equation and $\mathrm{Ma}$ and $\mathrm{Ge}$ [9] solved 3D convection diffusion equation.
Another practice of non-uniform grid discretization is space transformation. Ge and Zhang [10] solved singularly perturbed problems by discretizing the computational domain on a non-uniform grid to resolve the boundary layers, and then a grid transformation technique is used to map the non-uniform grid to a uniform one. The solution procedure of this method is complicated, expensive and sometimes error-prone.

In a departure from these two practices, a transformation-free HOC finite difference solution procedure was proposed for the steady 2D convection diffusion equation on non-uniform grid by Kalita et al. [11] Ge and Cao [12] developed a multigrid method based on [11] to solve the 2D convection diffusion equation. Recently, Ge et al. [13] proposed a multigrid method for solving the $3 \mathrm{D}$ Poisson equation, which is a convectiondiffusion equation with zero convection and constant diffusion coefficients.

This paper extends the work of Kalita et al. [11] to solve 3D convection diffusion equation with variable coefficients on a cubic non-uniform grid. Our approach is based on the use of Taylor series expansion, up to the fourth order terms, at a particular point with arbitrary mesh sizes in each of the three directions to approximate the derivatives appearing in the $3 \mathrm{D}$ convection diffusion equation. The original 
convection diffusion equation is then used again to replace the resulting higher order derivative terms. This leads to a higher order scheme on a compact stencil of nineteen points. The present scheme makes it possible to use whatever non-uniform pattern of spacing one chooses in either direction. We introduce an algebraic multigrid solver for the first time, to the best of our knowledge, as an attractive tool for solving the 3D convection diffusion problem on non-uniform grids with transformation-free HOC scheme.

The paper is organized in five sections. Section 2 presents the basic formulations and derivation of the proposed HOC scheme for variable convection coefficient case. Section 3 describes the Algebraic Multi-Grid (AMG) method. The numerical results for four boundary layers test cases are presented in Section 4. Finally, Section 5 contains the conclusions.

\section{Basic formulations and numerical procedure}

Consider the $3 \mathrm{D}$ convection diffusion equation in the form

$-\left(\frac{\partial^{2} u}{\partial x^{2}}+\frac{\partial^{2} u}{\partial y^{2}}+\frac{\partial^{2} u}{\partial z^{2}}\right)+p(x, y, z) \frac{\partial u}{\partial x}+q(x, y, z) \frac{\partial u}{\partial y}+r(x, y, z) \frac{\partial u}{\partial z}=f(x, y, z) \quad$ in $\Omega$

with proper Dirichlet boundary conditions on $\partial \Omega$. Here the coefficients $\mathrm{p}, \mathrm{q}, \mathrm{r}$ and forcing function $\mathrm{f}$, as well as the unknown function $\mathrm{u}$ are sufficiently smooth functions, and have the required continuous partial derivatives. $\Omega$ is a cubic region in $\Re^{3}$ defined by $\mathrm{a}_{1} \leq \mathrm{x} \leq \mathrm{a}_{2}, \mathrm{~b}_{1} \leq \mathrm{y} \leq \mathrm{b}_{2}$, $\mathrm{c}_{1} \leq \mathrm{z} \leq \mathrm{c}_{2}$.

The discretization is carried out on a non-uniform 3D grid. The intervals $\left[\mathrm{a}_{1}, \mathrm{a}_{2}\right],\left[\mathrm{b}_{1}, \mathrm{~b}_{2}\right]$ and $\left[\mathrm{c}_{1}, \mathrm{c}_{2}\right]$ are divided into sub-intervals by the points $\mathrm{a}_{1}=\mathrm{x}_{0}, \mathrm{x}_{1}, \mathrm{x}_{2}, \ldots, \mathrm{x}_{\mathrm{m}-1}, \mathrm{x}_{\mathrm{m}}=\mathrm{a}_{2} \quad, \quad \mathrm{~b}_{1}=$ $\mathrm{y}_{0}, \mathrm{y}_{1}, \mathrm{y}_{2}, \ldots, \mathrm{y}_{\mathrm{n}-1}, \mathrm{y}_{\mathrm{n}}=\mathrm{b}_{2}$

and $\mathrm{c}_{1}=\mathrm{z}_{0}, \mathrm{z}_{1}, \mathrm{z}_{2}, \ldots, \mathrm{z}_{\mathrm{k}-1}, \mathrm{z}_{\mathrm{k}}=\mathrm{c}_{2}$. In the $\mathrm{x}$-direction, the forward and backward step lengths are respectively given by $x_{\mathrm{f}}=\mathrm{x}_{\mathrm{i}+1}-\mathrm{x}_{\mathrm{i}}, \mathrm{x}_{\mathrm{b}}=\mathrm{x}_{\mathrm{i}}-\mathrm{x}_{\mathrm{i}-1}, 1 \leq \mathrm{i} \leq \mathrm{m}-1$ and in the $\mathrm{y}$ - and $\mathrm{z}$-directions, $\mathrm{y}_{\mathrm{f}}, \mathrm{y}_{\mathrm{b}}, \mathrm{z}_{\mathrm{f}}, \mathrm{z}_{\mathrm{b}}$ can be defined similarly.

For a function $\mathrm{u}(\mathrm{x}, \mathrm{y}, \mathrm{z})$ assumed smooth in the given domain, Taylor series expansion at point $(\mathrm{i}+1, \mathrm{j}, \mathrm{k})($ Fig. 1$)$ gives:

$u(i+1, j, k)=u_{i, j, k}+\left.x_{f} \frac{\partial u}{\partial x}\right|_{i j k}+\left.\frac{x_{f}^{2}}{2 !} \frac{\partial^{2} u}{\partial x^{2}}\right|_{i j k}+\left.\frac{x_{f}^{3}}{3 !} \frac{\partial^{3} u}{\partial x^{3}}\right|_{i j k}+\left.\frac{x_{f}^{4}}{4 !} \frac{\partial^{4} u}{\partial x^{4}}\right|_{i j k}+\left.\frac{x_{f}^{5}}{5 !} \frac{\partial^{5} u}{\partial x^{5}}\right|_{i j k}+O\left(x_{f}^{6}\right)$

Similarly at $(i-1, j, k)$

$u(i-1, j, k)=u_{i, j, k}-\left.x_{b} \frac{\partial u}{\partial x}\right|_{i j k}+\left.\frac{x_{b}^{2}}{2 !} \frac{\partial^{2} u}{\partial x^{2}}\right|_{i j k}-\left.\frac{x_{b}^{3}}{3 !} \frac{\partial^{3} u}{\partial x^{3}}\right|_{i j k}+\left.\frac{x_{b}^{4}}{4 !} \frac{\partial^{4} u}{\partial x^{4}}\right|_{i j k}-\left.\frac{x_{b}^{5}}{5 !} \frac{\partial^{5} u}{\partial x^{5}}\right|_{i j k}+O\left(x_{b}^{6}\right)$

From Eqs (2) and (3), we have

$$
\begin{aligned}
& \left.\frac{\partial u}{\partial x}\right|_{i j k}=\frac{u_{i+1, j, k}-u_{i-1, j, k}}{x_{f}+x_{b}}-\left.\frac{1}{2}\left(x_{f}-x_{b}\right) \frac{\partial^{2} u}{\partial x^{2}}\right|_{i j k}-\left.\frac{1}{6}\left(x_{f}^{2}+x_{b}^{2}-x_{f} x_{b}\right) \frac{\partial^{3} u}{\partial x^{3}}\right|_{i j k}-\left.\frac{1}{4 !}\left(x_{f}-x_{b}\right)\left(x_{f}^{2}+x_{b}^{2}\right) \frac{\partial^{4} u}{\partial x^{4}}\right|_{i j k}+O\left(\frac{x_{f}^{5}+x_{b}^{5}}{x_{f}+x_{b}}\right) \\
& \text { and } \\
& \left.\frac{\partial^{2} u}{\partial x^{2}}\right|_{i j k}=\frac{2}{x_{f}+x_{b}}\left[\frac{u_{i+1, j, k}}{x_{f}}+\frac{u_{i-1, j, k}}{x_{b}}-\left(\frac{1}{x_{f}}+\frac{1}{x_{b}}\right) u_{i, j, k}\right]-\left.\frac{1}{3}\left(x_{f}-x_{b}\right) \frac{\partial^{3} u}{\partial x^{3}}\right|_{i j k}-\left.\frac{1}{12}\left(x_{f}^{2}+x_{b}^{2}-x_{f} x_{b}\right) \frac{\partial^{4} u}{\partial x^{4}}\right|_{i j k}-\frac{1}{60}\left(x_{f}-x_{b}\right)\left(x_{f}^{2}+\right. \\
& \left.x_{b}^{2}\right)\left.\frac{\partial^{5} u}{\partial x^{5}}\right|_{i j k}+O\left(\frac{x_{f}^{5}+x_{b}^{5}}{x_{f}+x_{b}}\right)
\end{aligned}
$$



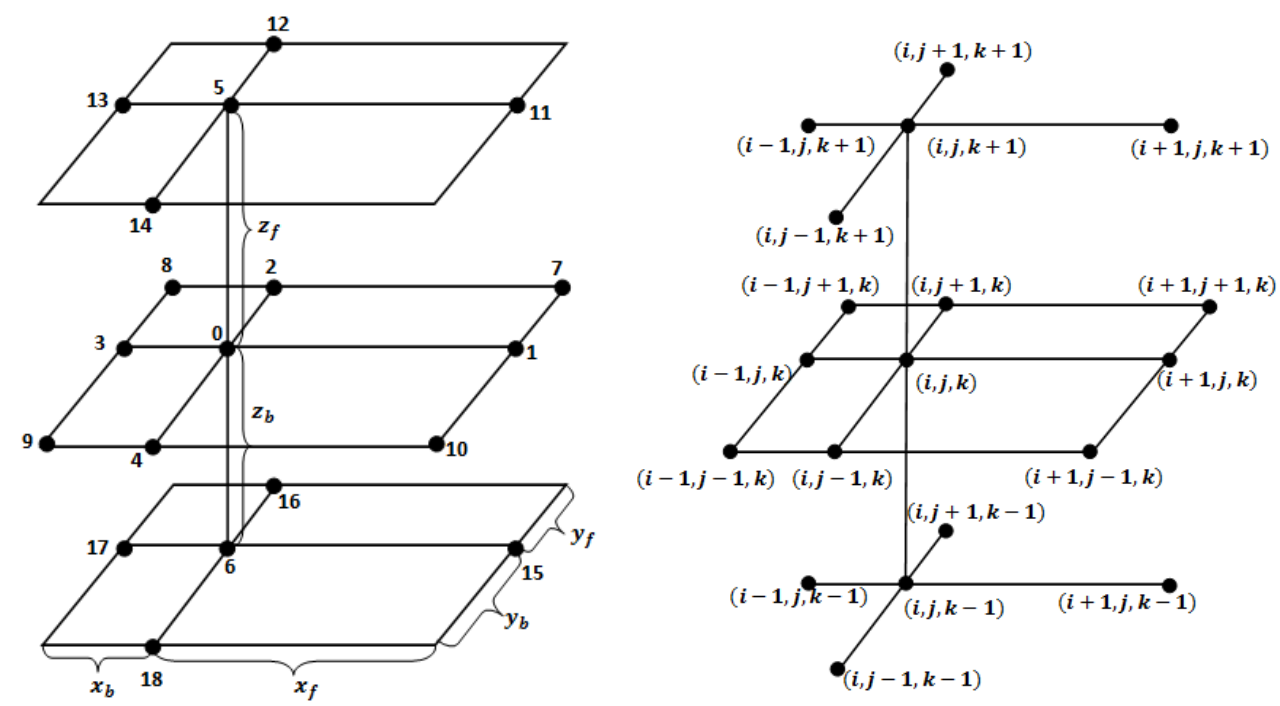

Figure 1. Non-uniform HOC stencil

In the $x$-direction, the first- and second-order central difference operators are defined by

$$
\delta_{x} u_{i j k}=\frac{u_{i+1, j, k}-u_{i-1, j, k}}{x_{f}+x_{b}} \text { and } \delta_{x}^{2} u_{i j k}=\frac{2}{x_{f}+x_{b}}\left[\frac{u_{i+1, j, k}}{x_{f}}+\frac{u_{i-1, j, k}}{x_{b}}-\left(\frac{1}{x_{f}}+\frac{1}{x_{b}}\right) u_{i, j, k}\right]
$$

With these notations, (5) becomes

$$
\left.\frac{\partial^{2} u}{\partial x^{2}}\right|_{i j k}=\delta_{x}^{2} u_{i j k}-\left.\frac{1}{3}\left(x_{f}-x_{b}\right) \frac{\partial^{3} u}{\partial x^{3}}\right|_{i j k}-\left.\frac{1}{12}\left(x_{f}^{2}+x_{b}^{2}-x_{f} x_{b}\right) \frac{\partial^{4} u}{\partial x^{4}}\right|_{i j k}-\left.\frac{1}{60}\left(x_{f}-x_{b}\right)\left(x_{f}^{2}+x_{b}^{2}\right) \frac{\partial^{5} u}{\partial x^{5}}\right|_{i j k}+O\left(\frac{x_{f}^{5}+x_{b}^{5}}{x_{f}+x_{b}}\right)
$$

From Eqs (4) and (6), the first derivative may be approximated as

$$
\left.\frac{\partial u}{\partial x}\right|_{i j k}=\delta_{x} u_{i j k}-\frac{1}{2}\left(x_{f}-x_{b}\right) \delta_{x}^{2} u_{i j k}-\left.\frac{x_{f} x_{b}}{6} \frac{\partial^{3} u}{\partial x^{3}}\right|_{i j k}-\left.\frac{1}{24} x_{f} x_{b}\left(x_{f}-x_{b}\right) \frac{\partial^{4} u}{\partial x^{4}}\right|_{i j k}+O\left(\frac{x_{f}^{5}+x_{b}^{5}}{x_{f}+x_{b}}\right)
$$

Similar expressions can be derived for the $y-$ and $z$-derivatives

$\left[-\delta_{x}^{2}-\delta_{y}^{2}-\delta_{z}^{2}+p\left\{\delta_{x}-0.5\left(x_{f}-x_{b}\right) \delta_{x}^{2}\right\}+q\left\{\delta_{y}-0.5\left(y_{f}-y_{b}\right) \delta_{y}^{2}\right\}+r\left\{\delta_{z}-0.5\left(z_{f}-z_{b}\right) \delta_{z}^{2}\right\}\right] u_{i j k}+\tau_{i j k}=$ $f_{i j k}$

where $\tau_{i j k}$ is given by

$\tau_{i j k}=H_{1} \frac{\partial^{3} u}{\partial x^{3}}+K_{1} \frac{\partial^{3} u}{\partial y^{3}}+L_{1} \frac{\partial^{3} u}{\partial z^{3}}+H_{2} \frac{\partial^{4} u}{\partial x^{4}}+K_{2} \frac{\partial^{4} u}{\partial y^{4}}+L_{2} \frac{\partial^{4} u}{\partial z^{4}}+\left(x_{f}-x_{b}\right)\left(x_{f}^{2}+x_{b}^{2}\right) \phi_{1}+\left(y_{f}-y_{b}\right)\left(y_{f}^{2}+y_{b}^{2}\right) \phi_{2}+$ $\left(z_{f}-z_{b}\right)\left(z_{f}^{2}+z_{b}^{2}\right) \phi_{3}+O\left(\frac{x_{f}^{5}+x_{b}^{5}}{x_{f}+x_{b}}\right)$

with $\phi_{1}, \phi_{2}$ and $\phi_{3}$ being the leading truncation error terms , where $\phi_{1}=\left.\frac{-1}{60} \frac{\partial^{5} u}{\partial x^{5}}\right|_{i j k}, \phi_{2}=\left.\frac{-1}{60} \frac{\partial^{5} u}{\partial y^{5}}\right|_{i j k}, \phi_{3}=\left.\frac{-1}{60} \frac{\partial^{5} u}{\partial z^{5}}\right|_{i j k}$ and

$H_{1}=\frac{1}{6}\left\{2\left(x_{f}-x_{b}\right)-p x_{f} x_{b}\right\}, \quad H_{2}=\frac{1}{24}\left\{2\left(x_{f}^{2}+x_{b}^{2}-x_{f} x_{b}\right)-\right.$ $\left.p x_{f} x_{b}\left(x_{f}-x_{b}\right)\right\}$
Now, we proceed to derive the HOC scheme for the 3D convection diffusion equation on non-uniform grids.

In view of Eqs (6) and (7), Eq. (1) may be approximated at the point $(\mathrm{i}, \mathrm{j}, \mathrm{k})$ as

$K_{1}=\frac{1}{6}\left\{2\left(y_{f}-y_{b}\right)-q y_{f} y_{b}\right\}, \quad K_{2}=\frac{1}{24}\left\{2\left(y_{f}^{2}+y_{b}^{2}-y_{f} y_{b}\right)-\right.$ $\left.q y_{f} y_{b}\left(y_{f}-y_{b}\right)\right\}$

and

$L_{1}=\frac{1}{6}\left\{2\left(z_{f}-z_{b}\right)-r z_{f} z_{b}\right\}, \quad L_{2}=\frac{1}{24}\left\{2\left(z_{f}^{2}+z_{b}^{2}-z_{f} z_{b}\right)-\right.$ $\left.r z_{f} z_{b}\left(z_{f}-z_{b}\right)\right\}$ 
It is important to note that, if $\tau_{\mathrm{ijk}}$ in Eq.(9) is approximated by the first six terms while neglecting the remaining terms then its truncation error is at least third order if the grid is nonuniform but fourth order for uniform grids.

\subsection{Derivation of $\mathrm{HOC}$}

The third- and fourth-order derivatives of $u$ in $\tau_{i j k}$ (Eq. (9)) are derived by differentiating the original Eq. (1) with respect to $\mathrm{x}, \mathrm{y}$ and $\mathrm{z}$. Using these derivatives, (9) can be written as

$$
\begin{aligned}
& \tau_{i j k}=\left(H_{1} p+H_{2} p^{2}+2 H_{2} \frac{\partial p}{\partial x}\right) \frac{\partial^{2} u}{\partial x^{2}}+\left(K_{1} q+K_{2} q^{2}+2 K_{2} \frac{\partial q}{\partial y}\right) \frac{\partial^{2} u}{\partial y^{2}}+\left(L_{1} r+L_{2} r^{2}+2 L_{2} \frac{\partial r}{\partial z}\right) \frac{\partial^{2} u}{\partial z^{2}}+\left\{\left(H_{1}+H_{2} p\right) \frac{\partial p}{\partial x}+\right. \\
& \left.\left(K_{1}+K_{2} q\right) \frac{\partial p}{\partial y}+\left(L_{1}+L_{2} r\right) \frac{\partial p}{\partial z}+H_{2} \frac{\partial^{2} p}{\partial x^{2}}+K_{2} \frac{\partial^{2} p}{\partial y^{2}}+L_{2} \frac{\partial^{2} p}{\partial z^{2}}\right\} \frac{\partial u}{\partial x}+\left\{\left(H_{1}+H_{2} p\right) \frac{\partial q}{\partial x}+\left(K_{1}+K_{2} q\right) \frac{\partial q}{\partial y}+\left(L_{1}+L_{2} r\right) \frac{\partial q}{\partial z}+\right. \\
& \left.H_{2} \frac{\partial^{2} q}{\partial x^{2}}+K_{2} \frac{\partial^{2} q}{\partial y^{2}}+L_{2} \frac{\partial^{2} q}{\partial z^{2}}\right\} \frac{\partial u}{\partial y}+\left\{\left(H_{1}+H_{2} p\right) \frac{\partial r}{\partial x}+\left(K_{1}+K_{2} q\right) \frac{\partial r}{\partial y}+\left(L_{1}+L_{2} r\right) \frac{\partial r}{\partial z}+H_{2} \frac{\partial^{2} r}{\partial x^{2}}+K_{2} \frac{\partial^{2} r}{\partial y^{2}}+L_{2} \frac{\partial^{2} r}{\partial z^{2}}\right\} \frac{\partial u}{\partial z}+ \\
& \left\{\left(H_{1}+H_{2} p\right) q+\left(K_{1}+K_{2} q\right) p+2 H_{2} \frac{\partial q}{\partial x}+2 K_{2} \frac{\partial p}{\partial y}\right\} \frac{\partial^{2} u}{\partial x \partial y}+\left\{\left(H_{1}+H_{2} p\right) r+\left(L_{1}+L_{2} r\right) p+2 H_{2} \frac{\partial r}{\partial x}+2 L_{2} \frac{\partial p}{\partial z}\right\} \frac{\partial^{2} u}{\partial x \partial z}+ \\
& \left\{\left(L_{1}+L_{2} r\right) q+\left(K_{1}+K_{2} q\right) r+2 L_{2} \frac{\partial q}{\partial z}+2 K_{2} \frac{\partial r}{\partial y}\right\} \frac{\partial^{2} u}{\partial y \partial z}-\left\{H_{1}+H_{2} p-K_{2} p\right\} \frac{\partial^{3} u}{\partial x \partial y^{2}}-\left\{K_{1}+K_{2} q-H_{2} q\right\} \frac{\partial^{3} u}{\partial x^{2} \partial y}- \\
& \left\{H_{1}+H_{2} p-L_{2} p\right\} \frac{\partial^{3} u}{\partial x \partial z^{2}}-\left\{L_{1}+L_{2} r-H_{2} r\right\} \frac{\partial^{3} u}{\partial x^{2} \partial z}-\left\{K_{1}+K_{2} q-L_{2} q\right\} \frac{\partial^{3} u}{\partial y \partial z^{2}}-\left\{L_{1}+L_{2} r-K_{2} r\right\} \frac{\partial^{3} u}{\partial y^{2} \partial z}-\left(H_{2}+\right. \\
& \left.K_{2}\right) \frac{\partial^{4} u}{\partial x^{2} \partial y^{2}}-\left(H_{2}+L_{2}\right) \frac{\partial^{4} u}{\partial x^{2} \partial z^{2}}-\left(K_{2}+L_{2}\right) \frac{\partial^{4} u}{\partial y^{2} \partial z^{2}}-\left\{\left(H_{1}+H_{2} p\right) \frac{\partial}{\partial x}+\left(K_{1}+K_{2} q\right) \frac{\partial}{\partial y}+\left(L_{1}+L_{2} r\right) \frac{\partial}{\partial z}+H_{2} \frac{\partial^{2}}{\partial x^{2}}+K_{2} \frac{\partial^{2}}{\partial y^{2}}+\right. \\
& \left.L_{2} \frac{\partial^{2}}{\partial z^{2}}\right\} f-\left(x_{f}-x_{b}\right)\left(x_{f}^{2}+x_{b}^{2}\right) \phi_{1}+\left(y_{f}-y_{b}\right)\left(y_{f}^{2}+y_{b}^{2}\right) \phi_{2}+\left(z_{f}-z_{b}\right)\left(z_{f}^{2}+z_{b}^{2}\right) \phi_{3}+O\left(\frac{x_{f}^{5}+x_{b}^{5}}{x_{f}+x_{b}}\right)
\end{aligned}
$$

From Eqs (8) and (10), we have the following HOC scheme on non-uniform grids for Eq. (1)

$\left[-A_{i j k} \delta_{x}^{2}-B_{i j k} \delta_{y}^{2}-C_{i j k} \delta_{z}^{2}+P_{i j k} \delta_{x}+Q_{i j k} \delta_{y}+R_{i j k} \delta_{z}+D_{i j k} \delta_{x} \delta_{y}+E_{i j k} \delta_{x} \delta_{z}+G_{i j k} \delta_{y} \delta_{z}-H_{i j k} \delta_{x} \delta_{y}^{2}-L_{i j k} \delta_{x}^{2} \delta_{y}-\right.$ $\left.M_{i j k} \delta_{x} \delta_{z}^{2}-N_{i j k} \delta_{x}^{2} \delta_{z}-O_{i j k} \delta_{y} \delta_{z}^{2}-S_{i j k} \delta_{y}^{2} \delta_{z}-T_{i j k} \delta_{x}^{2} \delta_{y}^{2}-V_{i j k} \delta_{x}^{2} \delta_{z}^{2}-W_{i j k} \delta_{y}^{2} \delta_{z}^{2}\right] u_{i j k}=F_{i j k}$

where the coefficients $A_{i j k}, B_{i j k}, \ldots, W_{i j k}$ are given by

$$
\begin{aligned}
& A_{i j k}=1-H_{2}\left(2 p_{x}+p^{2}\right)-H_{1} p+0.5\left(x_{f}-x_{b}\right) p \\
& B_{i j k}=1-K_{2}\left(2 q_{y}+q^{2}\right)-K_{1} q+0.5\left(y_{f}-y_{b}\right) q \\
& C_{i j k}=1-L_{2}\left(2 r_{z}+r^{2}\right)-L_{1} r+0.5\left(z_{f}-z_{b}\right) r \\
& P_{i j k}=p+H_{1} p_{x}+K_{1} p_{y}+L_{1} p_{z}+H_{2}\left(p p_{x}+p_{x x}\right)+K_{2}\left(q p_{y}+p_{y y}\right)+L_{2}\left(r p_{z}+p_{z z}\right) \\
& Q_{i j k}=q+H_{1} q_{x}+K_{1} q_{y}+L_{1} q_{z}+H_{2}\left(p q_{x}+q_{x x}\right)+K_{2}\left(q q_{y}+q_{y y}\right)+L_{2}\left(r q_{z}+q_{z z}\right) \\
& R_{i j k}=r+H_{1} r_{x}+K_{1} r_{y}+L_{1} r_{z}+H_{2}\left(p r_{x}+r_{x x}\right)+K_{2}\left(q r_{y}+r_{y y}\right)+L_{2}\left(r r_{z}+r_{z z}\right) \\
& D_{i j k}=H_{1} q+K_{1} p+H_{2}\left(p q+2 q_{x}\right)+K_{2}\left(p q+2 p_{y}\right) \\
& E_{i j k}=H_{1} r+L_{1} p+H_{2}\left(r p+2 r_{x}\right)+L_{2}\left(r p+2 p_{z}\right) \\
& M_{i j k}=H_{1}+p\left(H_{2}-L_{2}\right), N_{i j k}=L_{1}+r\left(L_{2}-H_{2}\right) \\
& O_{i j k}=K_{1}+q\left(K_{2}-L_{2}\right), S_{i j k}=L_{1}+r\left(L_{2}-K_{2}\right) \\
& T_{i j k}=H_{2}+K_{2}, V_{i j k}=H_{2}+L_{2}, W_{i j k}=K_{2}+L_{2}
\end{aligned}
$$

And

$F_{i j k}=f+\left(H_{1}+H_{2} p\right) f_{x}+\left(K_{1}+K_{2} q\right) f_{y}+\left(L_{1}+L_{2} r\right) f_{z}+H_{2} f_{x x}+K_{2} f_{y y}+L_{2} f_{z z}$

The expressions for $\tau_{i j k}$ in Eq. (10) clearly indicate that the local order of accuracy of the scheme is four or three depending upon the grid spacing. The order of the truncation error is four on uniform grids (when $\mathrm{x}_{\mathrm{f}}=\mathrm{x}_{\mathrm{b}}$, $\mathrm{y}_{\mathrm{f}}=\mathrm{y}_{\mathrm{b}}$ and $\mathrm{z}_{\mathrm{f}}=\mathrm{z}_{\mathrm{b}}$ ) and at least three when the grid spacing is non-uniform (when $\mathrm{x}_{\mathrm{f}} \neq \mathrm{x}_{\mathrm{b}}, \mathrm{y}_{\mathrm{f}} \neq \mathrm{y}_{\mathrm{b}}$ or $\mathrm{z}_{\mathrm{f}} \neq \mathrm{z}_{\mathrm{b}}$ ). In system of Eqs (12) and Eq. (13), we can use either exact derivatives or second order finite difference for the convection coefficients and the source term without reducing the order of approximation.

Substituting the finite difference formulas (see Appendix A) in Eq.(11) in view of the node numbering shown in Fig.(1), the 19 point high-order compact (HOC) scheme 
using non-uniform grids for the $3 \mathrm{D}$ convection diffusion equation (1) can be derived as follows

The coefficients $\alpha_{1}(l=0,1,2, \ldots, 18)$ are given as:

$$
\begin{aligned}
& \sum_{l=0}^{18} \alpha_{l} u_{l}=F_{i j k} \\
& \alpha_{0}=\frac{A_{i j k}}{h}\left(\frac{1}{x_{f}}+\frac{1}{x_{b}}\right)+\frac{B_{i j k}}{k}\left(\frac{1}{y_{f}}+\frac{1}{y_{b}}\right)+\frac{C_{i j k}}{l}\left(\frac{1}{z_{f}}+\frac{1}{z_{b}}\right)-\frac{4 T_{i j k}}{x_{f} x_{b} y_{f} y_{b}}-\frac{4 V_{i j k}}{x_{f} x_{b} z_{f} z_{b}}-\frac{4 W_{i j k}}{y_{f} y_{b} z_{f} z_{b}} \\
& \alpha_{1}=\frac{-A_{i j k}}{h x_{f}}+\frac{P_{i j k}}{2 h}+\frac{H_{i j k}}{2 h k}\left(\frac{1}{y_{f}}+\frac{1}{y_{b}}\right)+\frac{M_{i j k}}{2 h l}\left(\frac{1}{z_{f}}+\frac{1}{z_{b}}\right)+\frac{T_{i j k}}{h k}\left(\frac{1}{x_{f} y_{f}}+\frac{1}{x_{f} y_{b}}\right)+\frac{V_{i j k}}{h l}\left(\frac{1}{x_{f} z_{f}}+\frac{1}{x_{f} z_{b}}\right) \\
& \alpha_{2}=\frac{-B_{i j k}}{k y_{f}}+\frac{Q_{i j k}}{2 k}+\frac{L_{i j k}}{2 h k}\left(\frac{1}{x_{f}}+\frac{1}{x_{b}}\right)+\frac{o_{i j k}}{2 k l}\left(\frac{1}{z_{f}}+\frac{1}{z_{b}}\right)+\frac{T_{i j k}}{h k}\left(\frac{1}{x_{f} y_{f}}+\frac{1}{x_{b} y_{f}}\right)+\frac{W_{i j k}}{k l}\left(\frac{1}{y_{f} z_{f}}+\frac{1}{y_{f} z_{b}}\right) \\
& \alpha_{3}=\frac{-A_{i j k}}{h x_{b}}-\frac{P_{i j k}}{2 h}-\frac{H_{i j k}}{2 h k}\left(\frac{1}{y_{f}}+\frac{1}{y_{b}}\right)-\frac{M_{i j k}}{2 h l}\left(\frac{1}{z_{f}}+\frac{1}{z_{b}}\right)+\frac{T_{i j k}}{h k}\left(\frac{1}{x_{b} y_{f}}+\frac{1}{x_{b} y_{b}}\right)+\frac{V_{i j k}}{h l}\left(\frac{1}{x_{b} z_{f}}+\frac{1}{x_{b} z_{b}}\right) \\
& \alpha_{4}=\frac{-B_{i j k}}{k y_{b}}-\frac{Q_{i j k}}{2 k}-\frac{L_{i j k}}{2 h k}\left(\frac{1}{x_{f}}+\frac{1}{x_{b}}\right)-\frac{o_{i j k}}{2 k l}\left(\frac{1}{z_{f}}+\frac{1}{z_{b}}\right)+\frac{T_{i j k}}{h k}\left(\frac{1}{x_{f} y_{b}}+\frac{1}{x_{b} y_{b}}\right)+\frac{W_{i j k}}{k l}\left(\frac{1}{y_{b} z_{f}}+\frac{1}{y_{b} z_{b}}\right) \\
& \alpha_{5}=\frac{-C_{i j k}}{l z_{f}}+\frac{R_{i j k}}{2 l}+\frac{s_{i j k}}{2 k l}\left(\frac{1}{y_{f}}+\frac{1}{y_{b}}\right)+\frac{N_{i j k}}{2 h l}\left(\frac{1}{x_{f}}+\frac{1}{x_{b}}\right)+\frac{V_{i j k}}{h l}\left(\frac{1}{x_{f} z_{f}}+\frac{1}{x_{b} z_{f}}\right)+\frac{W_{i j k}}{k l}\left(\frac{1}{y_{f} z_{f}}+\frac{1}{y_{b} z_{f}}\right) \\
& \alpha_{6}=\frac{-C_{i j k}}{l z_{b}}-\frac{R_{i j k}}{2 l}-\frac{S_{i j k}}{2 k l}\left(\frac{1}{y_{f}}+\frac{1}{y_{b}}\right)-\frac{N_{i j k}}{2 h l}\left(\frac{1}{x_{f}}+\frac{1}{x_{b}}\right)+\frac{V_{i j k}}{h l}\left(\frac{1}{x_{f} z_{b}}+\frac{1}{x_{b} z_{b}}\right)+\frac{W_{i j k}}{k l}\left(\frac{1}{y_{f} z_{b}}+\frac{1}{y_{b} z_{b}}\right) \\
& \alpha_{7}=\frac{1}{4 h k}\left(D_{i j k}-2 \frac{L_{i j k}}{x_{f}}-2 \frac{H_{i j k}}{y_{f}}-4 \frac{T_{i j k}}{x_{f} y_{f}}\right), \alpha_{8}=\frac{1}{4 h k}\left(-D_{i j k}-2 \frac{L_{i j k}}{x_{b}}+2 \frac{H_{i j k}}{y_{f}}-4 \frac{T_{i j k}}{x_{b} y_{f}}\right) \\
& \alpha_{9}=\frac{1}{4 h k}\left(D_{i j k}+2 \frac{L_{i j k}}{x_{b}}+2 \frac{H_{i j k}}{y_{b}}-4 \frac{T_{i j k}}{x_{b} y_{b}}\right), \alpha_{10}=\frac{1}{4 h k}\left(-D_{i j k}+2 \frac{L_{i j k}}{x_{f}}-2 \frac{H_{i j k}}{y_{b}}-4 \frac{T_{i j k}}{x_{f} y_{b}}\right) \\
& \alpha_{11}=\frac{1}{4 h l}\left(E_{i j k}-2 \frac{M_{i j k}}{z_{f}}-2 \frac{N_{i j k}}{x_{f}}-4 \frac{V_{i j k}}{x_{f} z_{f}}\right), \alpha_{12}=\frac{1}{4 k l}\left(G_{i j k}-2 \frac{o_{i j k}}{z_{f}}-2 \frac{s_{i j k}}{y_{f}}-4 \frac{W_{i j k}}{y_{f} z_{f}}\right) \\
& \alpha_{13}=\frac{1}{4 h l}\left(-E_{i j k}+2 \frac{M_{i j k}}{z_{f}}-2 \frac{N_{i j k}}{x_{b}}-4 \frac{V_{i j k}}{x_{b} z_{f}}\right), \alpha_{14}=\frac{1}{4 k l}\left(-G_{i j k}+2 \frac{o_{i j k}}{z_{f}}-2 \frac{s_{i j k}}{y_{b}}-4 \frac{W_{i j k}}{y_{b} z_{f}}\right) \\
& \alpha_{15}=\frac{1}{4 h l}\left(-E_{i j k}-2 \frac{M_{i j k}}{z_{b}}+2 \frac{N_{i j k}}{x_{f}}-4 \frac{V_{i j k}}{x_{f} z_{b}}\right), \alpha_{16}=\frac{1}{4 k l}\left(-G_{i j k}-2 \frac{o_{i j k}}{z_{b}}+2 \frac{s_{i j k}}{y_{f}}-4 \frac{W_{i j k}}{y_{f} z_{b}}\right) \\
& \alpha_{17}=\frac{1}{4 h l}\left(E_{i j k}+2 \frac{M_{i j k}}{z_{b}}+2 \frac{N_{i j k}}{x_{b}}-4 \frac{V_{i j k}}{x_{b} z_{b}}\right), \alpha_{18}=\frac{1}{4 k l}\left(G_{i j k}+2 \frac{o_{i j k}}{z_{b}}+2 \frac{s_{i j k}}{y_{b}}-4 \frac{W_{i j k}}{y_{b} z_{b}}\right)
\end{aligned}
$$

and $F_{i j k}$ is given by Eq.(13).

The overall matrix and the source vector corresponding to the finite difference Eq. (14) are constructed using assembly process. The coefficients from $\alpha_{0}$ to $\alpha_{18}$ and $F_{i j k}$, are computed for all grid points according to the nodal stencil scheme shown in Fig. (1). Then after boundary conditions are incorporated, we obtain the system of linear equations $\mathrm{Au}=\mathrm{b}$.

\section{Algebraic Multigrid (AMG)}

The solution of the system of linear equations arising from the HOC scheme of $3 \mathrm{D}$ problems tends to be computationally intensive because it requires much more memory space and CPU time to obtain solutions with the desired accuracy. So, iterative solution methods are considered as the best choice rather than the direct methods in such situations. Multigrid methods (MG) [14-17] are among the most efficient iterative algorithms for solving linear systems associated with partial differential equations.
The basic idea of MG is to damp errors by utilizing multiple resolutions in an iterative scheme. Oscillatory components of the error are reduced through a smoothing procedure on a fine grid, while the smooth components are tackled using an auxiliary lower-resolution version of the problem (coarse grid). Two types of multigrid approaches may be distinguished: geometric multigrid (GMG) and algebraic multigrid (AMG) [18]. However, for convectiondominated problems, choice of the smoothing procedure and inter-grid operators are nontrivial for GMG method. Since a standard relaxation smoother may fail to achieve the optimal grid-independent convergence rate for solving convection diffusion equations with a high Reynolds number, the plane relaxation smoother or semi coarsening can be implemented to achieve better grid independency[1,6]. This requires special treatments of transfer operators and data structure.

On the other hand, for AMG, the coarsening process is fully automatic. Despite of the extra computation costs of 
this automation phase $[19,20]$, the most important strength of AMG is its flexibility and robustness in adapting itself to solve large classes of problems despite using very simple point-wise smoothers.

Thus, to solve the arising system, we choose to apply AMG. The efficiently implemented amg_grids_setup.m function by J. Boyle, D.J. Silvester [21] is used as a black box for construction of the coarser grids and computation of prolongation operators. This algorithm is based on [2224]. Our AMG method is based on the standard multigrid $\mathrm{V}$-Cycle. The V-Cycle is the computational process that goes from the fine grid down to the coarsest grid and then comes back from the coarsest grid up to the fine grid. We apply V $(0,2)$ cycles with a classical Gauss-Siedel smoother. The results of AMG solver is illustrated in Appendix B.

\section{Numerical Results}

Four test problems, with both constant and variable convection coefficients, are considered to demonstrate the accuracy of the present method. The first three of these problems are convection diffusion equations and the results of the present method on nonuniform grids are compared with those obtained using the most recent similar work which is available only for uniform grids [4-7]. For the purpose of comparison with 3D nonuniform grid, we consider the recent work of $\mathrm{Ge}$ et al. [13] that proposed a non-uniform HOC scheme to solve the 3D Poisson equation. So, our last test problem is a Poisson equation that can be obtained from Eq. (1) as a special case with zero convection coefficients.

The errors reported are the maximum absolute errors over the discretized grid. The accuracy order of a difference scheme is evaluated by the following formula Order $=\frac{\log \left(\mathrm{e}_{1} / \mathrm{e}_{2}\right)}{\log \left(\mathrm{N}_{2} / \mathrm{N}_{1}\right)}$

where $\mathrm{e}_{1}, \mathrm{e}_{2}$ are the maximum absolute errors for two different grids with $\left(\mathrm{N}_{1}+1\right)^{3}$ and $\left(\mathrm{N}_{2}+1\right)^{3}$ nodes, respectively.

The non-uniform grids are constructed easily. The interval $0 \leq x \leq 1$ can be divided uniformly into $i_{\max }$ intervals by nodes: $x_{i}=\frac{i}{i}, i=0,1, \cdots i_{\max }$. However a stretched grid can be obtained by:

$$
x_{i}=\frac{i}{i_{\max }}+\frac{\lambda_{x}}{\pi} \sin \left(\frac{\pi i}{i_{\text {max }}}\right), \quad i=0,1, \cdots i_{\max }
$$

where $\lambda_{\mathrm{x}}$ is a stretching parameter, $-1 \leq \lambda_{\mathrm{x}} \leq 1$. Similar grid stretching functions can be applied in $y-$ and $z$ directions.

\subsection{Problem 1}

Consider the following differential equation:

$$
-\epsilon\left(\mathrm{u}_{\mathrm{xx}}+\mathrm{u}_{\mathrm{yy}}+\mathrm{u}_{\mathrm{zz}}\right)+\frac{1}{1+\mathrm{y}} \mathrm{u}_{\mathrm{y}}=\mathrm{f}(\mathrm{x}, \mathrm{y}, \mathrm{z}), \quad 0 \leq
$$
$\mathrm{x}, \mathrm{y}, \mathrm{z} \leq 1$.

The Dirichlet boundary condition and source function $\mathrm{f}$ are determined such that the analytic solution is

$$
u(x, y, z)=z \cdot\left(e^{y-x}+2^{-1 / \epsilon}(1+y)^{1+1 / \epsilon}\right)
$$

This problem has a vertical boundary layer along $\mathrm{y}=1$. Therefore, a non-uniform grid along the $\mathrm{y}$-direction with clustering near $\mathrm{y}=1$ is used while keeping uniform grids along the $\mathrm{x}$ and $\mathrm{z}$ directions.

Table 1 gives the maximum absolute errors and the convergence order on uniform and non-uniform grids for $\epsilon=0.1,0.05$ and 0.01 . Although both uniform and nonuniform HOC produces the fourth order accuracy for this range of $\epsilon$, the values of errors are much less for the nonuniform scheme specially as $\epsilon$ decreases since the boundary

\begin{tabular}{|c|c|c|c|c|c|}
\hline & \multirow[t]{2}{*}{$\mathrm{N}$} & \multicolumn{2}{|c|}{ uniform } & \multicolumn{2}{|c|}{ Non-uniform } \\
\hline & & Error & Order & Error & Order \\
\hline & & & & $\lambda_{y}=0.1$ & \\
\hline & 17 & $5.89 \mathrm{E}-06$ & & $1.23 \mathrm{E}-06$ & \\
\hline \multirow[t]{4}{*}{$\epsilon=0.1$} & 33 & $3.67 \mathrm{E}-07$ & 4.00 & 7.57E-08 & 4.02 \\
\hline & 65 & $2.29 \mathrm{E}-08$ & 4.00 & 4.72E-09 & 4.00 \\
\hline & & & & $\lambda_{y}=0.2$ & \\
\hline & 17 & $1.25 \mathrm{E}-04$ & & $1.58 \mathrm{E}-05$ & \\
\hline \multirow[t]{4}{*}{$\epsilon=0.05$} & 33 & $7.95 \mathrm{E}-06$ & 3.97 & $9.37 \mathrm{E}-07$ & 4.08 \\
\hline & 65 & 4.95E-07 & 4.01 & $5.79 \mathrm{E}-08$ & 4.02 \\
\hline & & & & $\lambda_{y}=0.5$ & \\
\hline & 17 & $5.59 \mathrm{E}-02$ & & $1.24 \mathrm{E}-03$ & \\
\hline \multirow[t]{2}{*}{$\epsilon=0.01$} & 33 & $5.82 \mathrm{E}-03$ & 3.26 & $6.08 \mathrm{E}-05$ & 4.35 \\
\hline & 65 & $3.59 \mathrm{E}-04$ & 4.02 & 3.86E-06 & 3.98 \\
\hline
\end{tabular}
layer becomes more effective.

Table 1. Comparison of errors on uniform and non-uniform grids for Problem 1. 


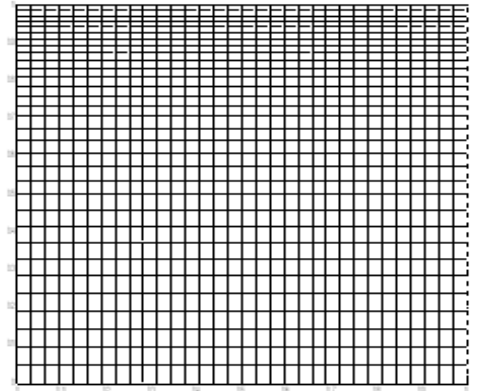

(a)

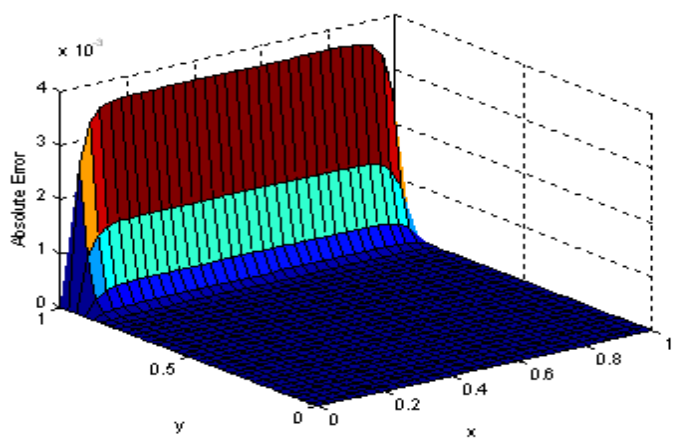

(c)

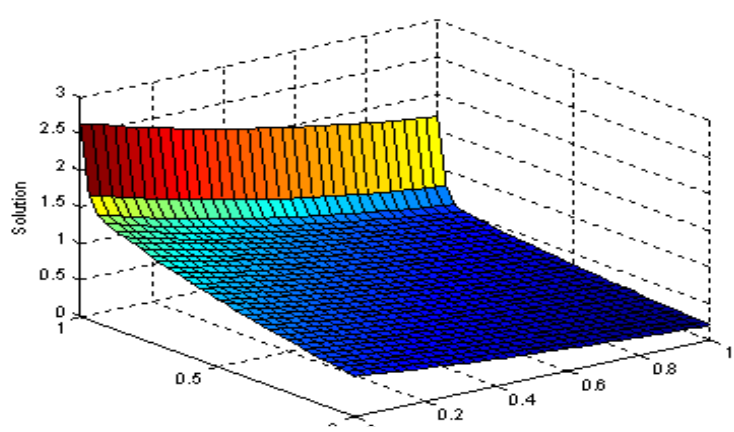

(b)

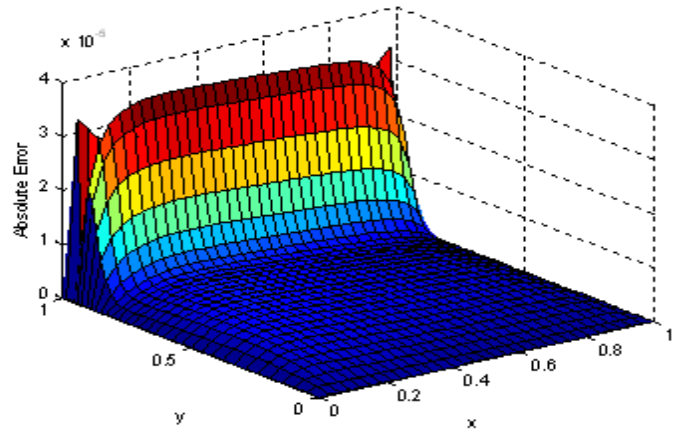

(d)

Figure 2. Results of Problem 1 for $\epsilon=0.01$ on grid $32^{3}$ in plane $z=0.5625$ (a) Non-uniform grid $\left(\lambda_{x}=\lambda_{z}=0, \lambda_{y}=0.55\right)$; (b) exact solution; (c) absolute error on uniform grid; (d) absolute error on non-uniform grid.

In order to demonstrate the efficiency of the proposed non-uniform $\mathrm{HOC}$, we plot results, at plane $\mathrm{z}=0.5625$ and for $\epsilon=0.01$, showing the exact solution in Fig. 2(b), the absolute error distribution on uniform grids in Fig. 2(c) and on non-uniform grids Fig. 2(d). We can see that the absolute error on non-uniform grids in the boundary layer is much smaller than that on uniform grids.

\subsection{Problem 2}

Consider Eq. (1) with: $p=-x(1-y)(2-z), q=$ $-y(1-z)(2-x), \quad r=-z(1-x)(2-y)$,

The boundary conditions and source function $f$ are given by the analytic solution.

$$
\mathrm{u}(\mathrm{x}, \mathrm{y}, \mathrm{z})=\frac{\mathrm{e}^{\mathrm{x} / \epsilon}+\mathrm{e}^{\mathrm{y} / \epsilon}+\mathrm{e}^{\mathrm{z} / \epsilon}-2}{\mathrm{e}^{1 / \epsilon}-1}
$$

Here the solution is almost zero everywhere except near $\mathrm{x}=1, \mathrm{y}=1$ and $\mathrm{z}=1$, where it has thin boundary layers. Most numerical methods have difficulty in accurately resolving the solution of such problems. To solve this problem, a non-uniform grid along the three directions with clustering near $\mathrm{x}=1, \mathrm{y}=1$ and $\mathrm{z}=1$ is used by suitable grid stretching parameters. For instance, when $\lambda_{x}=\lambda_{y}=$ $\lambda_{\mathrm{z}}=0.8$ the grid distribution in the xy-plane on grid $32^{3}$ is shown in Fig. 3(a).

Table 2 gives the maximum absolute errors and the convergence order on uniform and non-uniform grids.

We select different stretching parameters according to the value of $\epsilon$. As $\epsilon$ decreases, the boundary layer becomes thinner and nodes have to be more clustered to capture the singular behavior in the boundary layer. It can be observed that for $\epsilon=0.1$ and 0.05 , the computation on both uniform and non-uniform grids can keep fourth order convergence. But when $\epsilon$ decreases to 0.01 , the convergence rate on uniform grids decreases to third order while fourth order convergence still maintained on non-uniform grids. And the computation on non-uniform grids achieves significantly better accuracy than on uniform grids. In order to illustrate the accuracy of the proposed scheme, results of plane $\mathrm{z}=0.8125$,on uniform grid and plane $\mathrm{z}=0.8123$, on non-uniform grid, for $\epsilon=0.01$, are presented in Fig. 3 for the exact solution (b), the absolute error distribution on uniform grids (c), and on non-uniform grid (d). We can see that the absolute errors on non-uniform grids in the boundary layer are much smaller than that on uniform grids. 


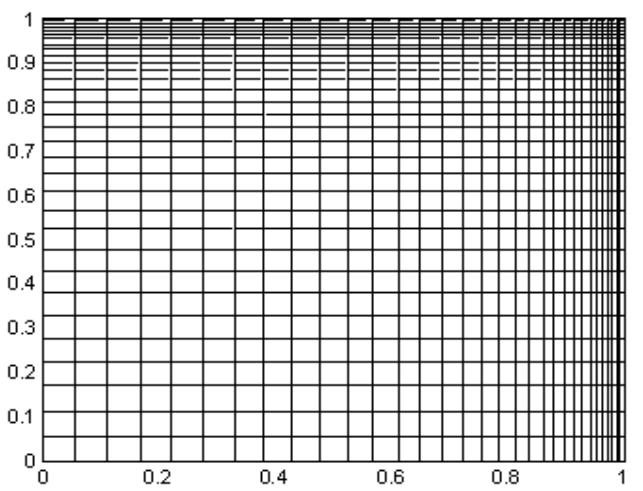

(a)

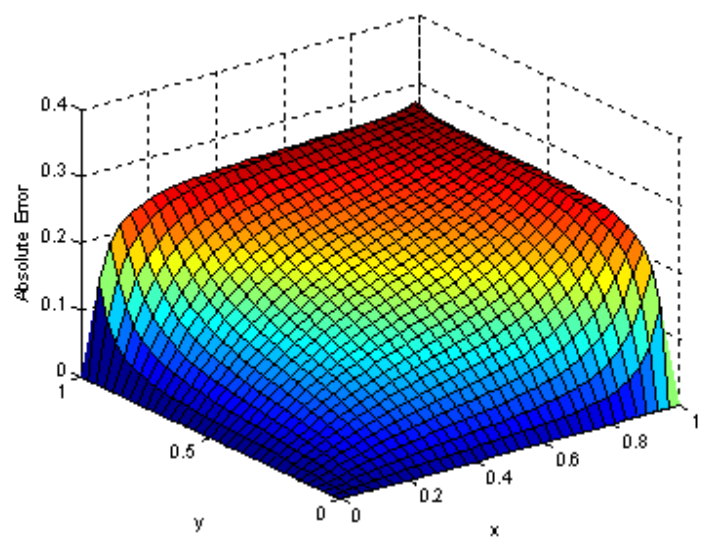

(c)

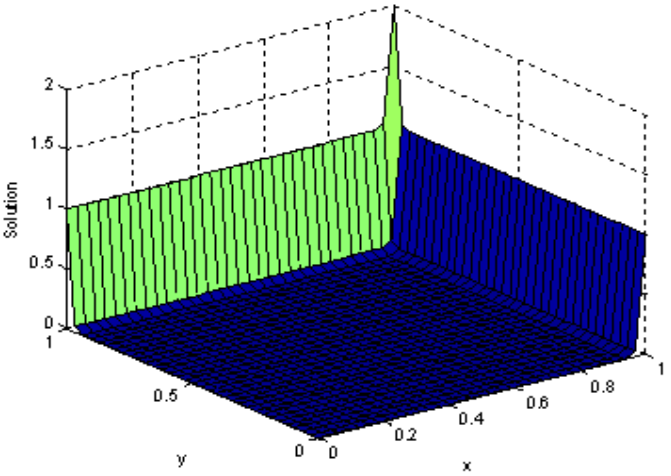

(b)

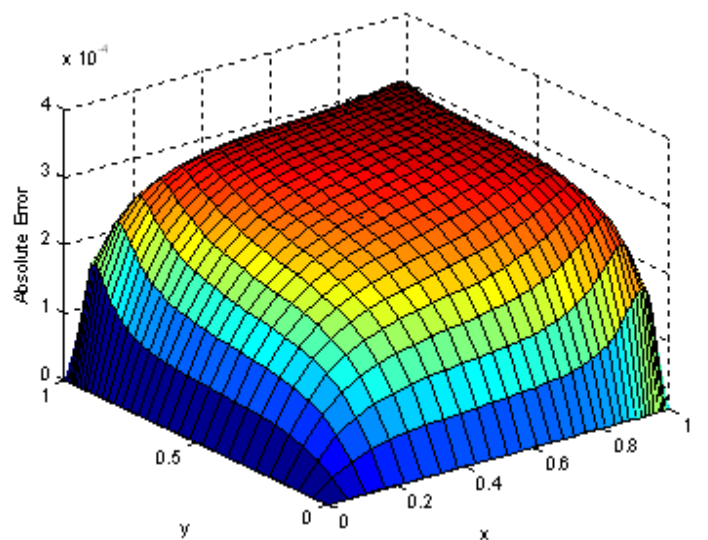

(d)

Figure 3. Results of Problem 2 at plane $z=0.8125$ (uniform grid) and plane $z=0.8123$ (non-uniform grid) for $\epsilon=0.01$ (a) Non-uniform grid $\left(\lambda_{x}=\lambda_{y}=\lambda_{z}=0.8,32^{3}\right)$, (b) exact solution, absolute error on (c) uniform grid, and (d) non-uniform grid.

Table 2. Comparison of errors on uniform and non-uniform grids for $\epsilon=0.1,0.05$ and 0.01

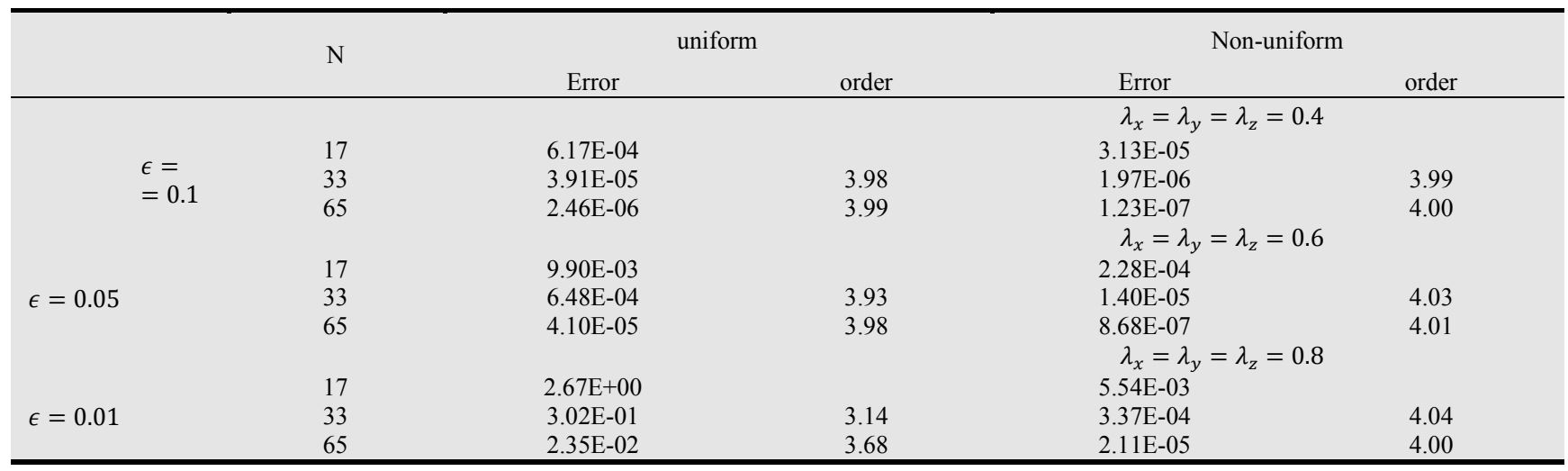

\subsection{Problem 3}

Consider the following differential equation:

$$
\begin{array}{r}
-\epsilon\left(u_{\mathrm{xx}}+\mathrm{u}_{\mathrm{yy}}+\mathrm{u}_{\mathrm{zz}}\right)+\mathrm{u}_{\mathrm{x}}+\mathrm{u}_{\mathrm{y}}+\mathrm{u}_{\mathrm{z}}= \\
=\mathrm{f}(\mathrm{x}, \mathrm{y}, \mathrm{z}), \quad 0 \leq \mathrm{x}, \mathrm{y}, \mathrm{z} \leq 1
\end{array}
$$

The Dirichlet boundary condition and source function $\mathrm{f}$ are determined such that the analytic solution is

$$
\mathrm{u}(\mathrm{x}, \mathrm{y}, \mathrm{z})=-\tanh (\mathrm{x} / 2 \epsilon) \tanh (\mathrm{y} / 2 \epsilon) \tanh (\mathrm{z} / 2 \epsilon)
$$

The solution has steep boundary layers along $\mathrm{x}=0, \mathrm{y}=$ 0 and $\mathrm{z}=0$. Therefore, a non-uniform grid along the three space directions with grid clustering near $\mathrm{x}=0, \mathrm{y}=0$ and $\mathrm{z}=0$ is used.

Fig. 4 depicts the grid distribution in the xy-plane when the mesh is $32^{3}, \lambda_{x}=\lambda_{y}=\lambda_{z}=-0.85 \quad$ (a). Next, the 
results for $\epsilon=0.01$ on plane $\mathrm{z}=0.1250$ are plotted: the exact solution (b), computed solution on uniform grid (c); computed solution on non-uniform grid (d); absolute error on uniform grids (e), and absolute error on non-uniform grids(f).

Table 3 gives the maximum absolute errors and the convergence order on uniform and non-uniform grids.

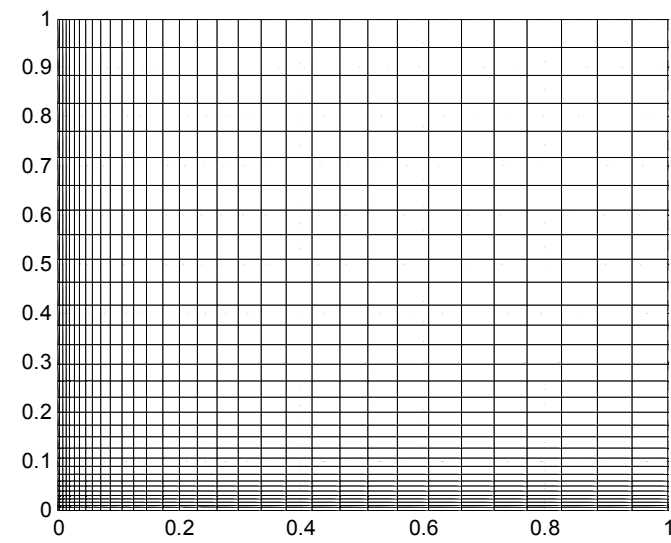

(a)

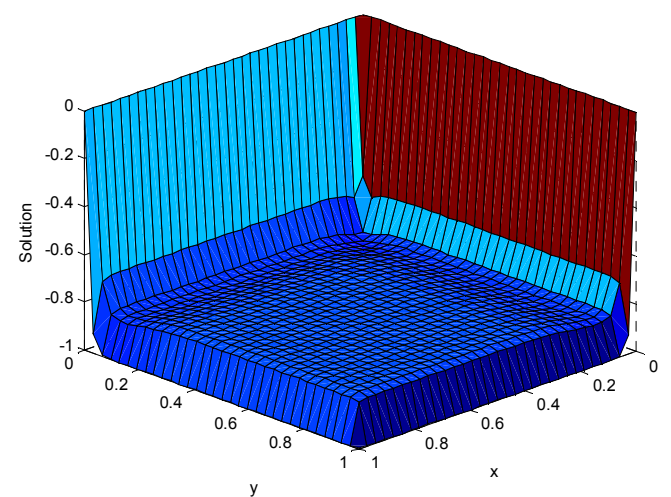

(c)

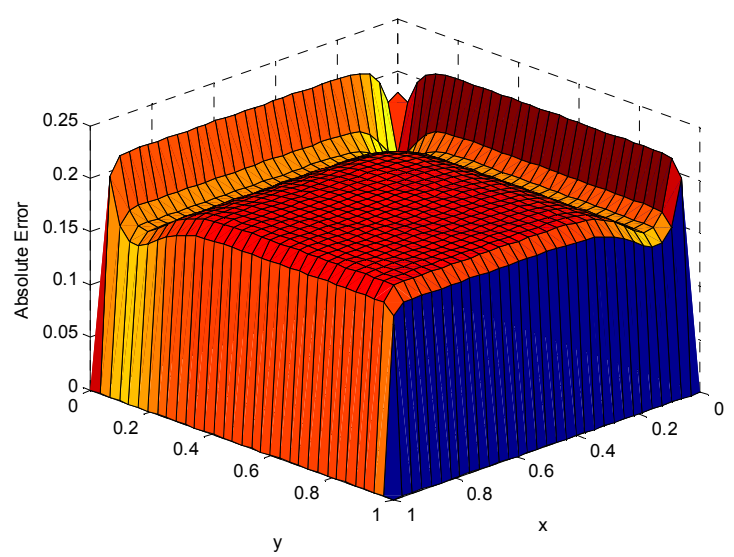

(e)
We can see that the computed accuracy on uniform grids deteriorates for $\epsilon=0.01$, a poor solution is obtained on uniform grids while considerably accurate solution is obtained and third or fourth order convergence is maintained for all values of $\epsilon$ on non-uniform grids.

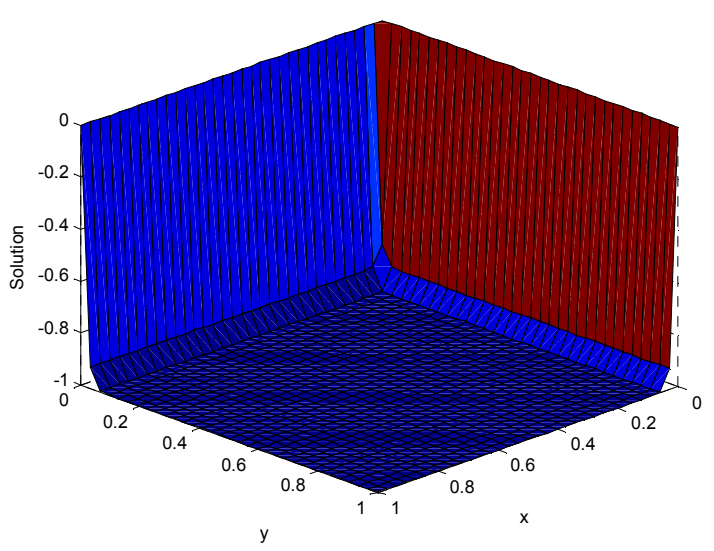

(b)

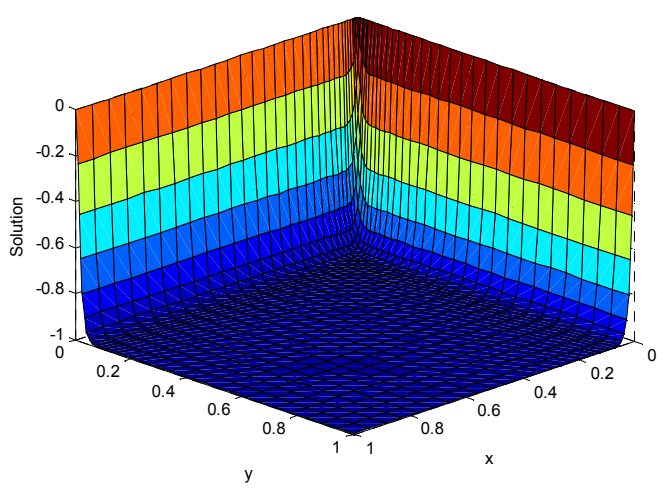

(d)

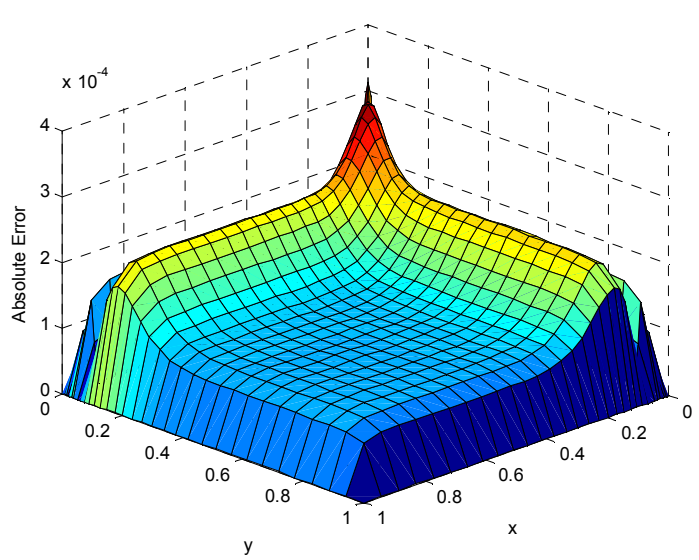

(f)

Figure 4. Results of Problem 3, $\epsilon=0.01$ on plane $z=0.125$ (a) Non-uniform grids $\left(\lambda_{x}=\lambda_{y}=\lambda_{z}=-0.85,32^{3}\right)$; (b) exact solution; (c) computed solution on uniform grid; (d) computed solution on non-uniform grid; (e) absolute error on uniform grids; $(f)$ absolute error on non-uniform grids. 
Table 3. Comparison of errors on uniform and non-uniform grids for Problem 3 for $\epsilon=0.1,0.05$ and 0.01

\begin{tabular}{|c|c|c|c|c|c|}
\hline & \multirow[t]{2}{*}{$\mathrm{N}$} & \multicolumn{2}{|c|}{ Uniform } & \multicolumn{2}{|c|}{ Non-uniform } \\
\hline & & Error & Order & Error & order \\
\hline \multirow{3}{*}{$\epsilon=0.1$} & 17 & $4.00 \mathrm{e}-04$ & \multicolumn{3}{|c|}{$\lambda_{x}=\lambda_{y}=\lambda_{z}=-0.5$} \\
\hline & 33 & $2.46 \mathrm{e}-05$ & 4.02 & $6.10 \mathrm{e}-06$ & 4.02 \\
\hline & 65 & $1.54 \mathrm{e}-06$ & 4.00 & $\begin{array}{r}3.81 \mathrm{e}-07 \\
\lambda_{x}=\lambda_{y}\end{array}$ & 4.00 \\
\hline \multirow{3}{*}{$\epsilon=0.05$} & 17 & $7.61 \mathrm{e}-03$ & & $4.52 \mathrm{e}-04$ & \\
\hline & 33 & $4.28 \mathrm{e}-04$ & 4.15 & $2.69 \mathrm{e}-05$ & 4.07 \\
\hline & 65 & $2.64 \mathrm{e}-05$ & 4.02 & $1.69 \mathrm{e}-06$ & 3.99 \\
\hline \multicolumn{6}{|c|}{$\lambda_{x}=\lambda_{y}=\lambda_{z}=-0.85$} \\
\hline \multirow{3}{*}{$\epsilon=0.01$} & 17 & $3.52 \mathrm{e}-01$ & & $6.57 \mathrm{e}-03$ & \\
\hline & 33 & $2.27 \mathrm{e}-01$ & 0.63 & $4.22 \mathrm{e}-04$ & 3.96 \\
\hline & 65 & $1.99 \mathrm{e}-02$ & 3.51 & $2.69 \mathrm{e}-05$ & 3.97 \\
\hline
\end{tabular}

\subsection{Problem 4}

For the purpose of comparison with existing numerical results, we consider the recent work by Ge et al. [13] that introduced HOC scheme for solving the 3D Poisson equation on non-uniform grids. In this problem, we consider the special case of the convection diffusion equation (1) when $p=q=r=0$ to reduce it to $3 D$ Poisson equation.

$$
-u_{x x}-u_{y y}-u_{z z}=f(x, y, z), \quad 0 \leq x, y, z \leq 1
$$

The Dirichlet boundary condition and source function $\mathrm{f}$ are determined such that the analytic solution is

$$
\mathrm{u}(\mathrm{x}, \mathrm{y}, \mathrm{z})=\frac{\left(1-\mathrm{e}^{(\mathrm{x}-1) / \epsilon}\right)\left(1-\mathrm{e}^{(\mathrm{y}-1) / \epsilon}\right)\left(1-\mathrm{e}^{(\mathrm{z}-1) / \epsilon}\right)}{\left(1-\mathrm{e}^{-1 / \epsilon}\right)^{3}}
$$

Here the exact solution has boundary layers along $\mathrm{x}=1$, $\mathrm{y}=1$ and $\mathrm{z}=1$. Therefore, a non-uniform grid along all three directions with clustering near $\mathrm{x}=1, \mathrm{y}=1$ and $\mathrm{z}=1$ is used.

When $\lambda_{\mathrm{x}}, \lambda_{\mathrm{y}}$ and $\lambda_{\mathrm{z}}$ are more close to 1 , more grid points are clustered near $\mathrm{x}=1, \mathrm{y}=1$ and $\mathrm{z}=1$. For $\lambda_{\mathrm{x}}=$ $\lambda_{\mathrm{y}}=\lambda_{\mathrm{z}}=0.8$ on grid $32^{3}$ and $\epsilon=0.01$, the grid in the $\mathrm{xy}$ plane is shown in Fig. 5(a). Results in plane $\mathrm{z}=$ 0.8125 (for uniform grid) and $\mathrm{z}=0.8123$ (for nonuniform grid) are shown in Figs. 5(b), (c), (d), (e) and (f) for: exact solution, computed solution on uniform grid, computed solution with the proposed scheme on nonuniform grid $\left(\lambda_{\mathrm{x}}=\lambda_{\mathrm{y}}=\lambda_{\mathrm{z}}=0.8\right)$, absolute error on uniform and non-uniform grids, respectively.

Table 4 gives the maximum absolute errors and the convergence order on uniform and non-uniform grids.

It is observed that the convergence on uniform grid cannot reach fourth order but non-uniform grid, with suitable stretching parameter $\lambda_{\mathrm{x}}=\lambda_{\mathrm{y}}=\lambda_{\mathrm{z}}=0.8$, is more accurate and fourth convergence order is achieved. The case when $\epsilon=0.01$ is recently solved by Ge et al. [13], the results in our present work is identical as in [13].

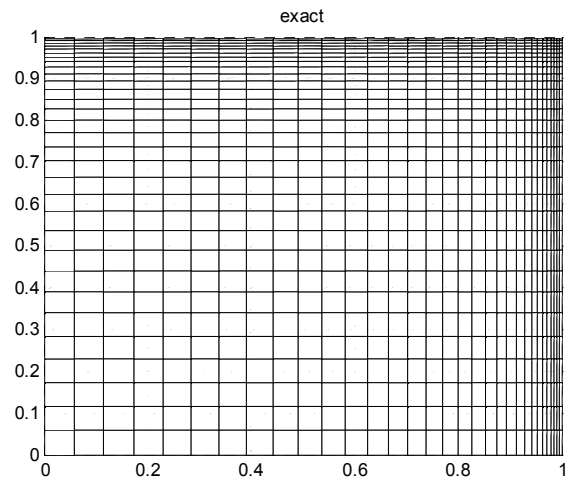

(a)

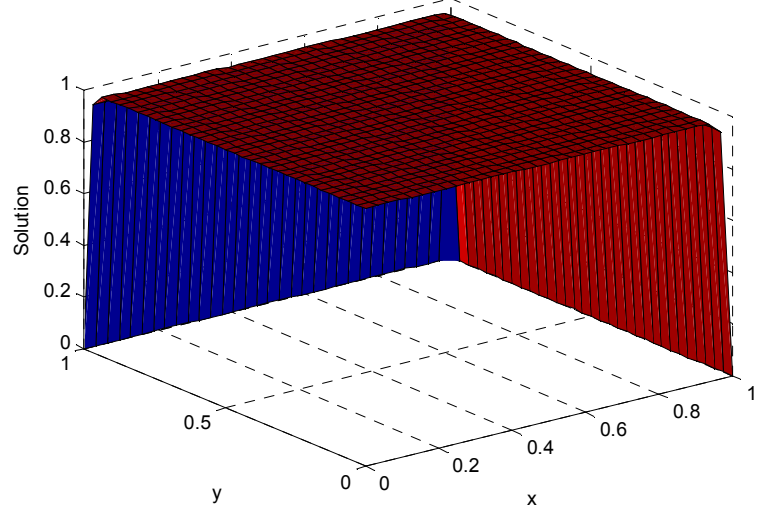

(b) 


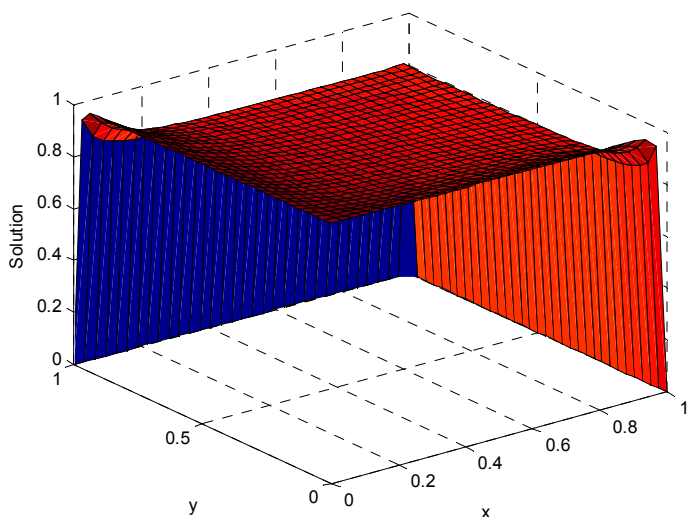

(c)

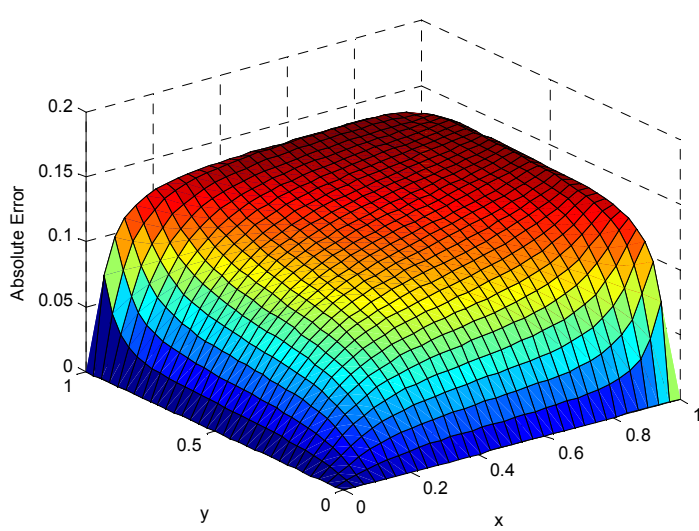

(e)

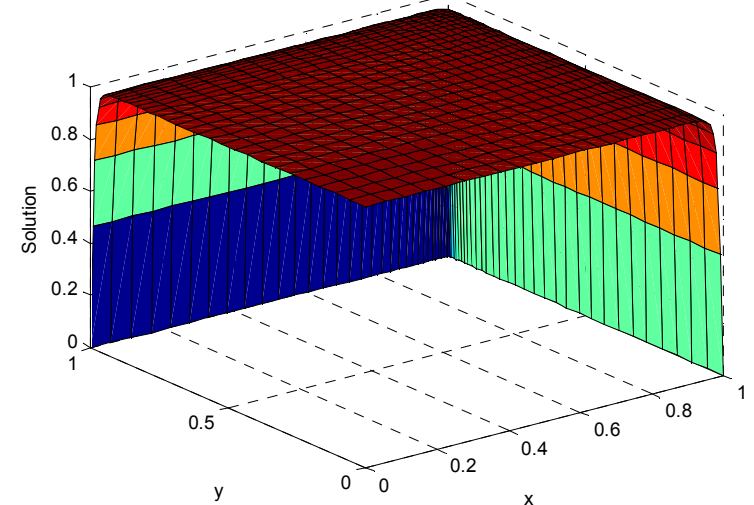

(d)

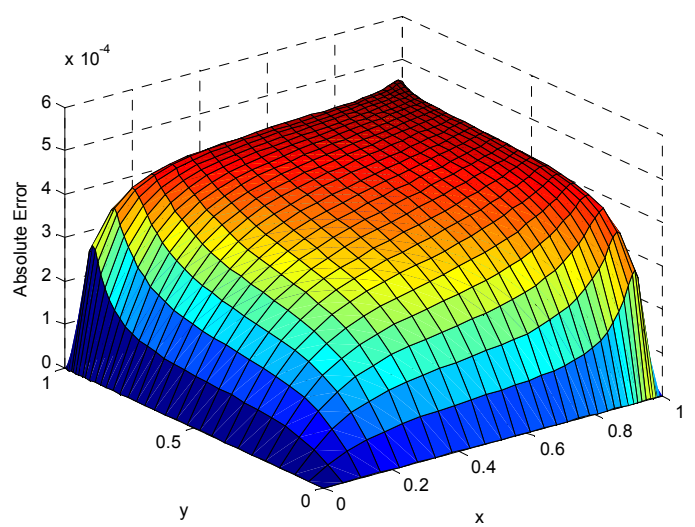

(f)

Figure 5. Results of Problem 4, on grid $32^{3}, \epsilon=0.01$ in the plane $z=0.8125$ (for uniform grid) $z=0.8123$ (for non-uniform grid) (a) Non-uniform grid $\left(\lambda_{x}=\lambda_{y}=\lambda_{z}=0.8\right.$ ); (b) exact solution; (c) computed solution on uniform grid; (d) computed solution on non-uniform grid; absolute error distribution (e) on uniform grids; and (f) on non-uniform grids.

Table 4. Comparison of errors on uniform and non-uniform grids for Problem 4.

\begin{tabular}{|c|c|c|c|c|c|}
\hline & \multirow[t]{2}{*}{$\mathrm{N}$} & \multicolumn{2}{|c|}{ Uniform } & \multicolumn{2}{|c|}{ Non-uniform } \\
\hline & & Error & Order & Error & order \\
\hline \multirow{3}{*}{$\epsilon=0.1$} & 17 & $3.28 \mathrm{e}-04$ & & $\begin{array}{c}\lambda_{x}=\lambda_{y} \\
5.05 \mathrm{e}-05\end{array}$ & \\
\hline & 33 & $2.13 e-05$ & 3.94 & $3.19 \mathrm{e}-06$ & 3.98 \\
\hline & 65 & $1.36 \mathrm{e}-06$ & 3.97 & $\begin{array}{r}2.00 \mathrm{e}-07 \\
\lambda_{x}=\lambda_{y}\end{array}$ & 4.00 \\
\hline \multirow{3}{*}{$\epsilon=0.05$} & 17 & $5.01 \mathrm{e}-03$ & & $3.60 \mathrm{e}-04$ & \\
\hline & 33 & $3.26 \mathrm{e}-04$ & 3.94 & $2.24 \mathrm{e}-05$ & 4.01 \\
\hline & 65 & $2.12 \mathrm{e}-05$ & 3.94 & $1.39 \mathrm{e}-06$ & 4.01 \\
\hline \multirow{5}{*}{$\epsilon=0.01$} & & & & $\lambda_{x}=\lambda_{y}$ & \\
\hline & 17 & $6.65 \mathrm{E}-01$ & & $8.46 \mathrm{e}-03$ & \\
\hline & 33 & $1.45 \mathrm{E}-01$ & 2.20 & $5.06 \mathrm{e}-04$ & 4.06 \\
\hline & 65 & $1.38 \mathrm{e}-02$ & 3.39 & $3.12 \mathrm{e}-05$ & 4.02 \\
\hline & & & & $\lambda_{x}=\lambda_{y}$ & \\
\hline \multirow{3}{*}{$\epsilon=0.001$} & 17 & $10 \mathrm{e}-01$ & & $2.48 \mathrm{e}-01$ & \\
\hline & 33 & $10 \mathrm{e}-01$ & 0.00 & $1.19 \mathrm{e}-02$ & 4.38 \\
\hline & 65 & $10 \mathrm{e}-01$ & 0.00 & $6.95 \mathrm{e}-04$ & 4.10 \\
\hline
\end{tabular}




\section{Conclusions}

We have proposed a transformation-free HOC finite difference scheme on non uniform grids for solving the 3D convection-diffusion equation. Generally, this scheme is third- to fourth- order accuracy and makes it possible to use whatever non-uniform pattern of spacing one chooses in either direction. Fourth-order accuracy, for boundary layer problems, is achieved on non-uniform grids with suitable stretching parameters as more grid points are clustered in the boundary layer. This scheme can solve 3D convectiondiffusion equation with constant, variable and zero (Poisson equation) convection coefficients. AMG method is applied to solve the resulting linear system from HOC scheme without need to specify special relaxation schemes and transfer operators as required in GMG method. Numerical results show that both uniform [4-7] and non- uniform HOC schemes produce very accurate solutions for smooth problems. But for boundary layer problems, the uniform HOC scheme gives poor solutions while nonuniform HOC scheme maintains accurate solutions.

The present method can be extended to solve other 3D partial differential equations, such as Navier-Stokes equations, problem involves Neumann boundary conditions as well as partial differential equations with irregular domains. The benefits of employing the HOC schemes and extrapolation can be also a future extension.

\section{Appendix A: Details of the finite difference operators}

The expressions for the finite difference operators appearing in Eq. (8) and (11) are as follows:

$$
\begin{aligned}
& \delta_{x} u_{i j k}=\frac{u_{i+1, j, k}-u_{i-1, j, k}}{2 h}, \delta_{y} u_{i j k}=\frac{u_{i, j+1, k}-u_{i, j-1, k}}{2 k}, \delta_{z} u_{i j k}=\frac{u_{i, j, k+1}-u_{i, j, k-1}}{2 l} \\
& \delta_{x}^{2} u_{i j k}=\frac{1}{h}\left\{\frac{u_{i+1, j, k}}{x_{f}}-\left(\frac{1}{x_{f}}+\frac{1}{x_{b}}\right) u_{i j k}-\frac{u_{i-1, j, k}}{x_{b}}\right\}, \delta_{y}^{2} u_{i j k}=\frac{1}{k}\left\{\frac{u_{i, j+1, k}}{y_{f}}-\left(\frac{1}{y_{f}}+\frac{1}{y_{b}}\right) u_{i j k}-\frac{u_{i, j-1, k}}{y_{b}}\right\} \\
& \delta_{z}^{2} u_{i j k}=\frac{1}{l}\left\{\frac{u_{i, j, k+1}}{z_{f}}-\left(\frac{1}{z_{f}}+\frac{1}{z_{b}}\right) u_{i j k}-\frac{u_{i, j, k-1}}{z_{b}}\right\} \\
& \delta_{x} \delta_{y} u_{i j k}=\frac{1}{4 h k}\left(u_{i+1, j+1, k}-u_{i+1, j-1, k}-u_{i-1, j+1, k}+u_{i-1, j-1, k}\right) \\
& \delta_{x} \delta_{z} u_{i j k}=\frac{1}{4 h l}\left(u_{i+1, j, k+1}-u_{i+1, j, k-1}-u_{i-1, j, k+1}+u_{i-1, j, k-1}\right) \\
& \delta_{y} \delta_{z} u_{i j k}=\frac{1}{4 k l}\left(u_{i, j+1, k+1}-u_{i, j+1, k-1}-u_{i, j-1, k+1}+u_{i, j-1, k-1}\right) \\
& \delta_{x} \delta_{y}^{2} u_{i j k}=\frac{1}{2 h k}\left\{\frac{1}{y_{f}}\left(u_{i+1, j+1, k}-u_{i-1, j+1, k}\right)-\left(\frac{1}{y_{f}}+\frac{1}{y_{b}}\right)\left(u_{i+1, j, k}-u_{i-1, j, k}\right)+\frac{1}{y_{b}}\left(u_{i+1, j-1, k}-u_{i-1, j-1, k}\right)\right\} \\
& \delta_{x}^{2} \delta_{y} u_{i j k}=\frac{1}{2 h k}\left\{\frac{1}{x_{f}}\left(u_{i+1, j+1, k}-u_{i+1, j-1, k}\right)-\left(\frac{1}{x_{f}}+\frac{1}{x_{b}}\right)\left(u_{i, j+1, k}-u_{i, j-1, k}\right)+\frac{1}{x_{b}}\left(u_{i-1, j+1, k}-u_{i-1, j-1, k}\right)\right\} \\
& \delta_{x} \delta_{z}^{2} u_{i j k}=\frac{1}{2 h l}\left\{\frac{1}{z_{f}}\left(u_{i+1, j, k+1}-u_{i-1, j, k+1}\right)-\left(\frac{1}{z_{f}}+\frac{1}{z_{b}}\right)\left(u_{i+1, j, k}-u_{i-1, j, k}\right)+\frac{1}{z_{b}}\left(u_{i+1, j, k-1}-u_{i-1, j, k-1}\right)\right\} \\
& \delta_{x}^{2} \delta_{z} u_{i j k}=\frac{1}{2 h l}\left\{\frac{1}{x_{f}}\left(u_{i+1, j, k+1}-u_{i+1, j, k-1}\right)-\left(\frac{1}{x_{f}}+\frac{1}{x_{b}}\right)\left(u_{i, j, k+1}-u_{i, j, k-1}\right)+\frac{1}{x_{b}}\left(u_{i-1, j, k+1}-u_{i-1, j, k-1}\right)\right\} \\
& \delta_{y} \delta_{z}^{2} u_{i j k}=\frac{1}{2 k l}\left\{\frac{1}{z_{f}}\left(u_{i, j+1, k+1}-u_{i, j-1, k+1}\right)-\left(\frac{1}{z_{f}}+\frac{1}{z_{b}}\right)\left(u_{i, j+1, k}-u_{i, j-1, k}\right)+\frac{1}{z_{b}}\left(u_{i, j+1, k-1}-u_{i, j-1, k-1}\right)\right\} \\
& \delta_{y}^{2} \delta_{z} u_{i j k}=\frac{1}{2 k l}\left\{\frac{1}{y_{f}}\left(u_{i, j+1, k+1}-u_{i, j+1, k-1}\right)-\left(\frac{1}{y_{f}}+\frac{1}{y_{b}}\right)\left(u_{i, j, k+1}-u_{i, j, k-1}\right)+\frac{1}{y_{b}}\left(u_{i, j-1, k+1}-u_{i, j-1, k-1}\right)\right\} \\
& \delta_{x}^{2} \delta_{y}^{2} u_{i j k}=\frac{1}{k h}\left\{\frac{u_{i+1, j+1, k}}{x_{f} y_{f}}+\frac{u_{i-1, j+1, k}}{x_{b} y_{f}}-\left(\frac{1}{x_{f} y_{f}}+\frac{1}{x_{b} y_{f}}\right) u_{i, j+1, k}-\left(\frac{1}{x_{f} y_{f}}+\frac{1}{x_{f} y_{b}}\right) u_{i+1, j, k}+\left(\frac{1}{x_{f} y_{f}}+\frac{1}{x_{f} y_{b}}+\frac{1}{x_{b} y_{f}}+\frac{1}{x_{b} y_{b}}\right) u_{i j k}-\right. \\
& \left.\left(\frac{1}{x_{f} y_{b}}+\frac{1}{x_{b} y_{b}}\right) u_{i, j-1, k}-\left(\frac{1}{x_{b} y_{f}}+\frac{1}{x_{b} y_{b}}\right) u_{i-1, j, k}+\frac{u_{i+1, j-1, k}}{x_{f} y_{b}}+\frac{u_{i-1, j-1, k}}{x_{b} y_{b}}\right\}
\end{aligned}
$$




$$
\begin{aligned}
& \delta_{x}^{2} \delta_{z}^{2} u_{i j k}=\frac{1}{h l}\left\{\frac{u_{i+1, j, k+1}}{x_{f} z_{f}}+\frac{u_{i-1, j, k+1}}{x_{b} z_{f}}-\left(\frac{1}{x_{f} z_{f}}+\frac{1}{x_{b} z_{f}}\right) u_{i, j, k+1}-\left(\frac{1}{x_{f} z_{f}}+\frac{1}{x_{f} z_{b}}\right) u_{i+1, j, k}+\left(\frac{1}{x_{f} z_{f}}+\frac{1}{x_{f} z_{b}}+\frac{1}{x_{b} z_{f}}+\frac{1}{x_{b} z_{b}}\right) u_{i j k}-\right. \\
& \left.\left(\frac{1}{x_{f} z_{b}}+\frac{1}{x_{b} z_{b}}\right) u_{i, j, k-1}-\left(\frac{1}{x_{b} z_{f}}+\frac{1}{x_{b} z_{b}}\right) u_{i-1, j, k}+\frac{u_{i+1, j, k-1}}{x_{f} z_{b}}+\frac{u_{i-1, j, k-1}}{x_{b} z_{b}}\right\} \\
& \delta_{y}^{2} \delta_{z}^{2} u_{i j k}=\frac{1}{k l}\left\{\frac{u_{i, j+1, k+1}}{y_{f} z_{f}}+\frac{u_{i, j-1, k+1}}{y_{b} z_{f}}-\left(\frac{1}{y_{f} z_{f}}+\frac{1}{y_{b} z_{f}}\right) u_{i, j, k+1}-\left(\frac{1}{y_{f} z_{f}}+\frac{1}{y_{f} z_{b}}\right) u_{i, j+1, k}+\left(\frac{1}{y_{f} z_{f}}+\frac{1}{y_{f} z_{b}}+\frac{1}{y_{b} z_{f}}+\frac{1}{y_{b} z_{b}}\right) u_{i j k}-\right. \\
& \left.\left(\frac{1}{y_{f} z_{b}}+\frac{1}{y_{b} z_{b}}\right) u_{i, j, k-1}-\left(\frac{1}{y_{b} z_{f}}+\frac{1}{y_{b} z_{b}}\right) u_{i, j-1, k}+\frac{u_{i, j+1, k-1}}{y_{f} z_{b}}+\frac{u_{i, j-1, k-1}}{y_{b} z_{b}}\right\}
\end{aligned}
$$

where $x_{f}, y_{f}, z_{f}, x_{b}, y_{b}$ and $z_{b}$ are defined in section 2 and $h=\left(x_{f}+x_{b}\right) / 2, k=\left(y_{f}+y_{b}\right) / 2$ and $l=\left(z_{f}+z_{b}\right) / 2$

\section{Appendix B: Results of AMG solver}

Table B1 shows the number of constructed AMG grid levels for $\epsilon=0.01$ on non-uniform grids for: problem 2, and 4 that represent problems with variable and zero convection coefficients, respectively.

Table (B1) Number of constructed AMG grid levels on different grids $17^{3}, 33^{3}$, and $65^{3}$.

\begin{tabular}{ccc}
\hline $\mathrm{N}$ & \multicolumn{2}{c}{ AMG grid levels non-uniform } \\
Problem 2 & Problem 4 \\
\hline 17 & 11 & 12 \\
33 & 13 & 13 \\
65 & 17 & 17 \\
\hline
\end{tabular}

The convergence behavior (Residual Norm versus AMG-V(0,2)-Cycles) for problems 2 and 4 are shown in Figs. (B1 and B2), respectively. It is concluded that good convergence rates with slight dependence on mesh sizes is satisfactory even for the non-uniform grids and presence of boundary layers.

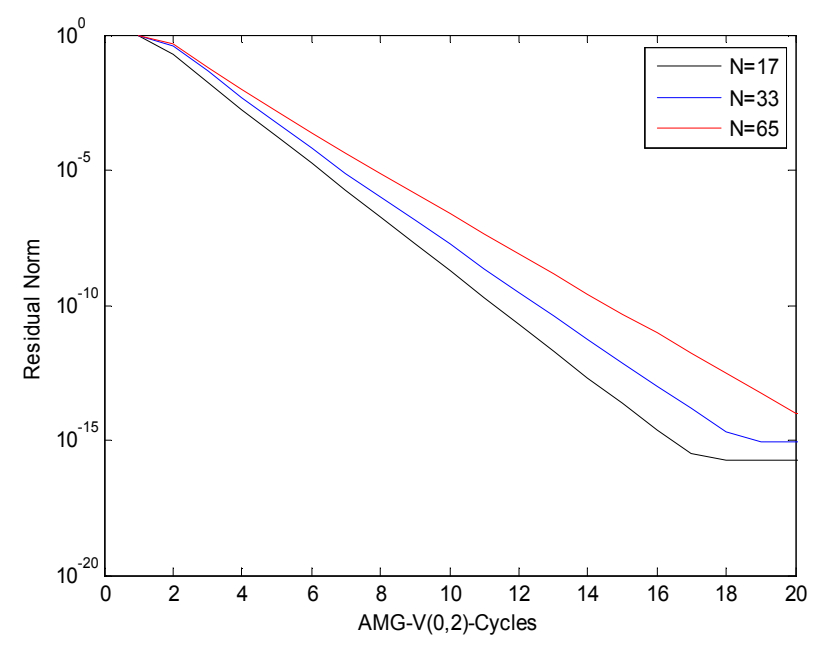

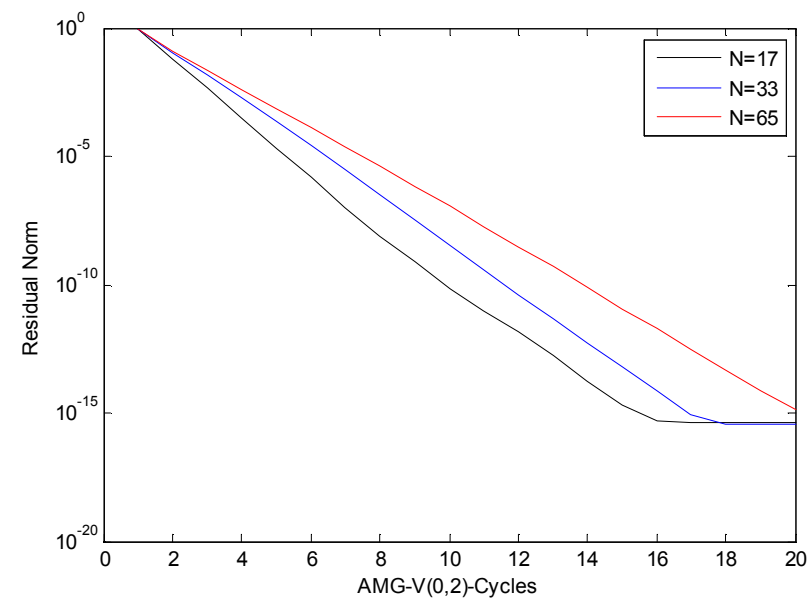

(B1)

(B2)

Figure (B1) and (B2): $A M G$ Convergence for Problem 2 and 4

\section{References}

[1] S. KARAA, J. Zhang, Convergence and Performance of Iterative Methods for Solving Variable Coefficient Convection-Diffusion Equation with a Fourth-Order Compact Difference Scheme , Computers and Mathematics with Applications 44 (2002) 457-479.

[2] J. Zhang, Numerical Simulation of 2D Square Driven Cavity Using Fourth-Order Compact Finite Difference Schemes, Computers and Mathematics with Applications 45 (2003) 43-52.

[3] Y. Wang, J. Zhang, Integrated fast and high accuracy computation of convection diffusion equations using multiscale multigrid method, Numerical Methods for Partial Differential Equations, 27(2):399-414, 2011

[4] L. Ge, J. Zhang, Symbolic computation of high order compact difference for three dimensional linear elliptic partial differential equations with variable schemes coefficients, Journal of Computational and Applied Mathematics 143 (2002) 9-27

[5] M. gupta, J. zhang, High accuracy multigrid solution of the 3D Convection Diffusion equation, Applied Mathematics and Computation 113 (2000) 249-274

[6] Y. Wang, J. Zhang, Fast and robust sixth-order multigrid computation for the three-dimensional Convection- 
Diffusion equation, Journal of Computational and Applied Mathematics 234, pp.3496-3506, 2010.

[7] J. Zhang, An explicit fourth-order compact finite difference scheme for three-dimensional Convection-Diffusion equation, Communications in Numerical Methods in Engineering, Vol. 14, 263--280 (1998).

[8] Y. Ge, Multigrid method and fourth-order compact difference discretization scheme with unequal meshsizes for 3D poisson equation, Journal of Computational Physics 229 (2010) 6381-6391

[9] Y. Ma, Y. Ge, A high order finite difference method with Richardson extrapolation for 3D Convection Diffusion equation, Applied Mathematics and Computation 215 (2010) 3408-3417

[10] L. Ge, J, Zhang, High Accuracy Iterative Solution of Convection Diffusion Equation with Boundary Layers on Non-uniform Grids, Journal of Computational Physics171,560-578 (2001)

[11] J. Kalita, A. Dass, D. Dalal, A transformation-free HOC scheme for steady Convection-Diffusion on non-uniform grids, Int. J. Numer. Meth. Fluids 44 (2004) 33-53

[12] Y. Ge, F. Cao, Multigrid method based on the transformation-free HOC scheme on non-uniform grids for 2D Convection Diffusion problems, Journal of Computational Physics 230 (2011) 4051-4070

[13] Y. Ge, F. Cao, J. Zhang, A transformation-free HOC scheme and multigrid method for solving the 3D Poisson equation on non-uniform grids, Journal of Computational Physics (2012)

[14] A. Brandt, Multilevel Adaptive Solutions to Boundary Value
Problems, Mathematics of Computation, Vol.31, pp. 333390, 1977.

[15] W. Hachbusch, V. Trottenberg, Multigrid Methods Lecture Notes in Mathematics, Vol. 960, Springer: Berlin, 1982.

[16] W. L. Briggs, A Multigrid Tutorial, Society for Industrial and Applied Mathematics, Philadelphia, Pennsylvania, 1987.

[17] P. Wesseling, An Introduction to Multigrid Methods, Weily, England, 1992.

[18] K. Stuben, A Review of Algebraic Multigrid, Journal of Computational and Applied Mathematics, Vol.128, pp.281$309,2001$.

[19] C. Tien Wu and H. C. Elman, Analysis and Comparison of Geometric and Algebraic Multigrid for Convection-Diffusion Equations, SIAM J. Sci. Comput., Vol.28, pp.2208-2228, 2006.

[20] V. Gravemeier, M. W. Gee and W.A. Wall, "An algebraic variational multiscale-multigrid method based on plain aggregation for convection-diffusion problems", Comput. Methods Appl. Mech. Engrg. Vol.198, pp.3821-3835, 2009.

[21] www.maths.manchester.ac.uk/ gjs/ifiss

[22] W. Briggs, V. Henson, and S. McCormick, A Multigrid Tutorial, SIAM, Philadelphia, 2000. [Chapter 8: Algebraic Multigrid (AMG)]

[23] J. Ruge and K. Stuben, Algebraic multigrid (AMG), in Multigrid Methods, Frontiers in Applied Mathematics, S. F. McCormick, ed., SIAM, Philadelphia, 1987, pp. 73-130.

[24] U. Trottenberg, C. Oosterlee, and A. Schuller, Multigrid, Academic Press, London, 2001. [appendix A: Algebraic Multigrid (AMG) by K. Stuben] 